\title{
Digital Intra-Oral Scanner Impression in Implant Dentistry
}

\author{
Mohammed Hussein M Alsharbaty
}

Department of Prosthodontics, School of Dentistry, Tehran University of Medical Sciences (IC-TUMS), Tehran, Iran

Corresponding author: Mohammed Hussein M Alsharbaty, Department of Prosthodontics, School of Dentistry, Tehran University of Medical Sciences (IC-TUMS), Tehran, Iran, Tel: 989196539029; E-mail: mh-mohammedhussein@razi.tums.ac.ir

Received: 23 May 2017; Accepted: 30 May 2017; Published: 05 June 2017

Citation: Alsharbaty MH. Digital Intra-Oral Scanner Impression in Implant Dentistry. Ann Clin Lab Res. 2017; 5: 2.

\section{Opinion}

The first critical step in implant prostheses fabrication is making accurate impressions. The establishments of good fitting prostheses are determined at impression stage. During implant impressions making, the concentration is to accurately reproduce the implant location in 3-dimensions in relation to other intra-oral hard/soft tissue structures. Impression accuracy could affect the passive fit of framework construction, which affects the definitive outcome.

Over the past few years, digital technologies have advanced rapidly. This obviously influenced existing transformations in the science, industry, and daily life style. Together with gradual changes in three-dimensional technologies, new innovations also emerged in dental medicine. Starting with the first computer-aided approach in restorative dentistry in the late 1980s, enhancements in data achievement, processing, and manufacturing currently provide much faster and more accurate final results. The introduction and development of various intraoral scanners (IOSs) led to their popularity and broad application in dentistry.

Digital impression procedures may be an approach to improve the accuracy of implant-supported restorations, as by their nature, these prostheses eliminate the error-prone conventional impression and gypsum model casting, and warrant a high degree of standardization. There are various digital intra-oral scanners, which have developed to make direct impression from the oral cavity.

Digital impressions could improve patient acceptance, reduce possible distortion of impression materials, and allow for three-dimensional visualization. However, digital impression requires better soft tissue management, dry working field, and certainly necessitates a high learning curve. In the implant field, digital impressions would allow virtual assessments of the implant prosthetic space, depth of the restoration interface, and the emergence profile configuration before proceeding with the laboratory steps. Nevertheless, the discernable characteristics of digital impression, it requires more time to be clinically evaluated particularly in implant dentistry.

Theoretically, the digital impression approach let the clinician to avoid some of the hitches associated with the conventional approach, and could thus result in a more accurate implant impression. The accuracy of a digital impression depends not only on the accuracy of the intra-oral scanner, but also on the accuracy of fit of the scan body, the digital software, or the cast fabrication and implant analogue transfer to the casts, if the working casts fabricated. Intra- oral scanners build 3D models by combining several 3D images made of the same area from different angulations. This composition of different 3D sections can lead to registration errors; scan accuracy relies on both the scanning technology and the registration algorithm for the compilation of the final data.

Digital intra-oral implant impressions have presented achievement in the dental market, and with further development exhibit potential to offer possible alternative assistances to the clinician, the patient and the laboratory technician. Recent trends in the dental field suggest that digital impression is going to be gradually significant in the digital workflow for the fabrication of implant-supported restorations. It is important to assume in mind that prosthodontic reconstruction will remain a multi-step discipline requiring careful attention and accuracy at each step including intra-oral impressions. With regards to implant impressions, clinicians must accept that absolute accuracy remains an elusive goal regardless of any technique used. Therefore, clinicians must carefully evaluate the inherent error in any impression technique used and ultimately understand the clinical significance of any such error on an individual basis. 